Proceedings of the International Symposium on Physics of Materials (ISPMA 14), September 10-15, 2017, Prague

\title{
Micro-Scale Model of Thermomechanics in Solidifying Saturated Porous Media
}

\author{
A. Ž̇́K* AND M. BENEŠ \\ Department of Mathematics, Faculty of Nuclear Sciences and Physical Engineering, \\ Czech Technical University in Prague, Trojanova 13, Praha, 120 00, Czech Republic
}

\begin{abstract}
This article analyses processes accompanying solidification of a liquid-saturated porous medium by means of thermomechanical balance and admitting difference in density of liquid and solid phases. Complexity of these processes is given by the inherent heterogeneity of the volume occupied both by grains and pores of the medium. To understand details, we consider elementary pore configuration at the micro-scale. We have developed a micro-scale model describing mechanical, thermal, and phase change processes within a porous medium. The model provides spatio-temporal dependences of key variables and allows to evaluate the stresses caused by the difference in the specific volumes of the present phases.
\end{abstract}

DOI: 10.12693/APhysPolA.134.678

PACS/topics: 02.60.Lj, 47.55.N-, 64.70.D-, 62.20.--x

\section{Introduction}

The research is motivated by phenomena accompanying seasonal or climate changes affecting the upper layers of soil ground, by phenomena studied in planetary science in the context of deep structures of planetary interior, but also by demands of production of the high-tech composite materials applicable in technology or medicine (see e.g. [1-3]). Impact of it can be seen in better understanding climate changes, release of green-house gases as well as in the progress in high technology.

The process of porous medium solidification consists of several crucial phenomena involving bulk material changes, effects due to the pore structure and curvature of pore volume, and interactions of components. Although there are several macro-scale models, especially of soil freezing phenomenon [4-7], they are usually insufficiently general and complex, or single-purpose, or are too simple. Majority of attention in the problem modeling has been devoted to the frost heave [8], a phenomenon occurring due to seasonal or climate changes heaving heavy loads and causing damages in constructions.

Volumetric change of a liquid constituent of a porous medium is one of phenomena occurring within the medium when subjected to freezing conditions. Due to generic inhomogeneity of solidifying porous medium, its contribution to the structural response is rather approximated or empirically estimated. In order to understand modeling of this particular phenomenon, we are concerned with the phase transition in the context of a general volume shape at the micro-scale.

We present the micro-scale model of a solidifying porous medium that provides spatio-temporal dependences of key variables and allows to evaluate the surface

*corresponding author; e-mail: alexandr.zak@fjfi.cvut.cz forces exerted on grains caused by the change in the specific volume of the solidifying component as well as the resultant strains of the material.

Thus one of the aims of this contribution is to improve the understanding of details of the process at the microscale (see [9-11]). This is achieved by introducing a new approach to tracking the solid phase position as described in Sect. 2.3.

\section{Micro-scale model}

In this section, we describe our two-dimensional thermo-mechanical model of solidification within a liquid-saturated porous medium at the micro-scale capturing the density change. Due to the mechanical balance as well as changes of structural properties of the considered material the model is formulated in the Lagrangian coordinates. The position of solid phase is captured by means of the phase field method. The model is formulated under the following assumptions. A representative porous volume is a three-phase system consisting of colloidal solid particles surrounded solely by a phase-changing medium. The characteristic length of the particles and pores ranges from $1 \mu \mathrm{m}$ up to $1 \mathrm{~mm}$. The affiliation of quantities to a particular phase is expressed by indices as follows: $i$ for solid, $l$ for liquid, and $s$ for skeleton particles. The modeling concept is similar to the classical nucleation theory established by Gibbs [12]. Each individual phase component of the considered porous medium is assumed to be a continuum neighboring to the other phases. The processes are considered as quasi-static.

\subsection{Fluid-structure interaction}

Deformation of the domains occupied by particular phases is characterized by the displacement of all their material points. Let $\boldsymbol{X}$ denote the position of a material point at the reference configuration. Then its position change at moment $t$ can be expressed as $\boldsymbol{X}+\boldsymbol{u}(\boldsymbol{X}, t)$, 
where $\boldsymbol{u}$ is the displacement vector. Under the assumption of small deformation, the local force balance law for a phase $j \in\{i, l, s\}$ at the time $t$ and the point $\boldsymbol{X}$ of its domain is expressed as

$$
\varrho_{j} \frac{\partial^{2} \boldsymbol{u}_{j}}{\partial t^{2}}=\nabla \cdot \boldsymbol{\sigma}_{j}+\varrho_{j} f_{j}
$$

where $\varrho_{j}$ is the phase volumetric mass density, $\boldsymbol{\sigma}_{j}$ is the stress tensor, $f_{j}$ is the exterior force density, and $\nabla$ is the formal derivative vector with respect to the components of $\boldsymbol{X}$. We assume that solid phases behave like a linear elastic material. Thus their stress tensors can be related to the small deformation tensor $\hat{e}$ via the relations

$$
\boldsymbol{\sigma}_{j}=\boldsymbol{c}_{j} \hat{e}\left(\boldsymbol{u}_{j}\right), \quad \hat{e}\left(\boldsymbol{u}_{j}\right)=\frac{1}{2}\left(\nabla \boldsymbol{u}_{j}+\left(\nabla \boldsymbol{u}_{j}\right)^{\mathrm{T}}\right),
$$

where $\boldsymbol{c}$ is the elastic tensor. Assuming further that solids are homogeneous and isotropic, the elastic tensors can be fully characterized by two material parameters of the phase $j$, e.g. by the Young modulus $E_{j}$ and by the Poisson ratio $\nu_{j}$. Denoting the unit tensor by $\hat{I}$, Eq. (2.2) becomes

$\boldsymbol{\sigma}_{j}=\frac{E_{j}}{\left(1+\nu_{j}\right)} \hat{e}\left(\boldsymbol{u}_{j}\right)+\frac{\nu_{j} E_{j} \nabla \cdot \boldsymbol{u}_{j}}{\left(1+\nu_{j}\right)\left(1-2 \nu_{j}\right)} \hat{I}, j \in\{i, s\}$.

The liquid phase $(j=l)$ is assumed to act as a slightly compressible viscous Newtonian fluid. Such a material can be characterized with respect to the Lagrangian coordinates by the stress tensor in the form

$$
\boldsymbol{\sigma}_{l}=-p \hat{I}+\mu\left(\nabla \boldsymbol{v}+(\nabla \boldsymbol{v})^{\mathrm{T}}\right),
$$

where $p$ is the pressure, $\mu$ is the dynamic viscosity, and $\boldsymbol{v}=\partial \boldsymbol{u} / \partial t$ is the Lagrangian velocity. The momentum balance equation for the liquid phase is supplemented (like in [13]) with the equation of continuity for a slightly compressible fluid that reads

$$
\frac{1}{\varrho_{l} E_{l}} p+\nabla \cdot \boldsymbol{u}=0
$$

At phase interfaces, the balance conditions of corresponding quantities are described, in general, by the nonlinear equations coupling the states of neighboring phases (for details, see $[14,15]$ ). Applying the small-strain assumption and using the linearized forms, in agreement with [13], the coupling of the mechanical states expresses the continuity of velocity and balance of normal forces.

At the intersection of more phase interfaces, an additional condition is imposed. At equilibrium, the Young law is commonly used to determine the contact angle of the interfaces with respect to their surface energies. In dynamical situation, the no-slip condition leads to nonintegrable flow singularities at the contact of lines [16], indicating that such a model should be improved. Thus the flow conditions at the interface need to be reconsidered more in detail, as indicated in [17], or the flow near the contact lines needs to be remodelled.

\subsection{Heat transfer}

Heat balances in individual phases as well as on their mutual boundaries are expressed by means of temperature $T=T(\boldsymbol{X}, t)$. Since the considered system is subject of small deformations, the heat balance is linearized and formulated within the Lagrangian framework as well.
Assuming the phase $j$ with the specific heat capacity $c_{j}$ being equipped with the instantaneous heat flux density $q_{j}$, its heat equation can be formulated as

$$
\varrho_{j} c_{j} \frac{\partial T}{\partial t}=-\nabla \cdot q_{j}
$$

The heat transport through mutual boundaries is given by the continuity of the temperature and by conditions for the heat flux. At the grain-solid and grain-liquid interfaces, the normal heat fluxes from both phases are equal. The liquid-solid interface $\Gamma$ presents the place of the phase change of the single material, and thus the corresponding heat flux balance incorporates the latent heat of the phase transition. Since the effect of the interfacial energy is assumed to be small (as in [18]) compared to the latent heat, it can be neglected in the interfacial balance consideration. The flux condition at the liquid-solid interface is then given by the Stefan condition,

$$
\left[q_{i}-q_{l}\right] \cdot \boldsymbol{n}=\varrho_{i} l_{M}\left(v_{\Gamma}-\boldsymbol{v} \cdot \boldsymbol{n}\right),
$$

where $\boldsymbol{n}$ is the normal vector of the interface, $l_{\mathrm{M}}$ is the specific latent heat, and $v_{\Gamma}$ denotes the normal velocity of the interface.

The heat flux density $q_{j}$ can be given by the linearized Fourier law, which reads

$$
q_{j}=-k_{j} \nabla T_{j},
$$

where $k_{j}$ is the thermal conductivity of phase $j$.

Assuming the effects of the pressure and the interfacial entropy are small, a local dynamical equilibrium at the phase interface can be expressed by the Gibbs-Thomson equation for undercooling of $\Gamma$ (e.g. [19]),

$$
\varrho_{i} l_{M} \frac{T_{M}-T}{T_{M}}=\gamma\left(\kappa+\alpha v_{\Gamma}\right)
$$

where $T_{\mathrm{M}}$ is the bulk freezing point, $\gamma$ is the surface energy, $\kappa$ is the curvature of the interface, and $\alpha$ is the attachment kinetics coefficient.

\subsection{Interface modeling}

The Gibbs-Thomson formula (2.9) is related to the motion of solid-liquid interface $\Gamma$. In this article, we newly introduce a tool for the implicit tracking of the solid-liquid interface located in pores of a porous medium structure. This tool explores the diffuse-interface method based on the Cahn-Hilliard theory of phase transitions [20, 21]. For this purpose, we add a nonlinear partial differential equation known as the Allen-Cahn equation to the model, which produces a function $w$ describing the presence of phases at a given position and time. For more details, see [21-23].

This function $w=w(\boldsymbol{X}, t)$ (the order parameter) is expected to be near 0 , if the system is locally in the liquid state, and near 1 , if it is locally in the solid state. The transition from one to another state should take place in a thin transition layer. The interface $\Gamma$ is then identified by the level set $w=1 / 2$.

The Allen-Cahn equation is coupled to the heat balance, and together they form a system of phase-field equations in the Lagrangian coordinates. 


$$
\begin{aligned}
& \varrho c \frac{\partial T}{\partial t}=\nabla(k \nabla T)+\varrho_{i} l_{M} \frac{\partial w}{\partial t}, \\
& \alpha \xi^{2} \frac{\partial w}{\partial t}=\xi^{2} \nabla^{2} w+a w(w-1 / 2)(1-w)+f, \\
& f=\xi^{2} b \beta\left(T_{M}-T\right)|\nabla w|,
\end{aligned}
$$

where $\varrho, c, k$ are material characteristics, $a, b, \beta$ are positive constants, and $\xi>0$ is a small parameter, which is related to the transition-layer thickness.

The equations in various generalized setting were studied in, e.g. [22, 23] and in references therein.

The matched asymptotics can be used (e.g. as in [24]) to recover of the Stefan condition and the GibbsThomson law at the phase interface.

\subsection{Model equations}

Due to the introduction of the order parameter by the phase field method, the localization of individual phases is conveniently resolved, and definitions of the effective values of material parameters are allowed. The effective values can be related to the current phase content combining the corresponding bulk values as follows:

$$
\begin{aligned}
& \varrho=w \varrho_{i}+(1-w) \varrho_{l}, \quad c=w c_{i}+(1-w) c_{l}, \\
& k=w k_{i}+(1-w) k_{l} .
\end{aligned}
$$

The stress characteristic within the pore material can be expressed as

$$
\boldsymbol{\sigma}=w \boldsymbol{\sigma}_{i}+(1-w) \boldsymbol{\sigma}_{l}+w \boldsymbol{\psi}, \quad \boldsymbol{\psi}=\frac{\beta_{i} E_{i}}{1-2 \nu_{i}} \hat{I} .
$$

Tensor $\boldsymbol{\psi}$ represents the additional stresses in solid phase due to the structural change during the phase transition and can be characterized by $\beta_{i}$, the volume expansion ratio of the solid phase.

The evolution of the coordinate system is given by the Navier equations for the force balance.

Denoting the pore domain by $\Omega_{p}$, the skeleton domain by $\Omega_{s}$, and their inner mutual boundary by $\Gamma_{p s}$, the model equations read for $t>0$ as

$$
\begin{aligned}
& \varrho c \frac{\partial T_{p}}{\partial t}-\varrho_{i} l_{M} \frac{\partial w}{\partial t}=\nabla \cdot\left(k \nabla T_{p}\right) \quad \text { in } \Omega_{p} \\
& \alpha \xi^{2} \frac{\partial w}{\partial t}=\xi^{2} \nabla^{2} w+a w(w-1 / 2)(1-w)+f \quad \text { in } \Omega_{p} \\
& \varrho \frac{\partial^{2} \boldsymbol{u}_{p}}{\partial t^{2}}=\nabla \cdot \boldsymbol{\sigma} \quad \text { in } \Omega_{p} \\
& \frac{p}{\varrho_{l} E_{l}}+\nabla \cdot \boldsymbol{u}_{p}=0 ; f=\xi^{2} b \beta\left(T_{M}-T_{p}\right)|\nabla w| \text { in } \Omega_{p} \\
& \varrho_{s} c_{s} \frac{\partial T_{s}}{\partial t}=\nabla \cdot\left(k_{s} \nabla T_{s}\right) ; \varrho_{s} \frac{\partial^{2} \boldsymbol{u}_{s}}{\partial t^{2}}=\nabla \cdot \boldsymbol{\sigma}_{\boldsymbol{s}} \quad \text { in } \Omega_{s} \\
& T_{s}=T_{p} ; \boldsymbol{u}_{s}=\boldsymbol{u}_{p} \quad \text { on } \Gamma_{p s}, \\
& k_{s} \nabla T_{s} \cdot \boldsymbol{n}=k \nabla T_{p} \cdot \boldsymbol{n} ; \boldsymbol{\sigma}_{s} \boldsymbol{n}=\boldsymbol{\sigma} \boldsymbol{n} \text { on } \Gamma_{p s} \\
& w=0 ; \nabla w \cdot \boldsymbol{n}=0 \text { on } \Gamma_{p s} .
\end{aligned}
$$

Conditions (2.22) for the order parameter imply a permanent presence of a thin layer of the liquid phase along the pore boundaries, which, however, is in agreement with real world observations (e.g. [25]). Since the quantitative capturing of this phenomenon is not currently in the scope of this paper, the form of the conditions is considered appropriate. An ensuing modeling consequence is that the included boundary value problem (2.16) and (2.22) allows to model the dynamics of the solid-liquid transition neighboring with the another nonmelting phase and thus to overcome the sharp-interface formulation difficulties near a triple junction as mentioned in the end of Sect. 2.1.

The complete mathematical model which can give some information on solidifying dynamics in porous media at the micro-scale is supplemented by appropriate boundary conditions along the outer boundaries and an initial condition.

\section{Computational results}

In this section, we consider a liquid-saturated sample of porous medium. The computational domain presents a vertical cross-section through the medium and is composed of a cluster of grains surrounded by a liquid (see Fig. 1). The sample is subjected to a uniform thermal vertical gradient, and its boundaries are assumed not to be movable except the upper boundary in all directions and the left boundary in upwards. The material characteristics were used as in [11].

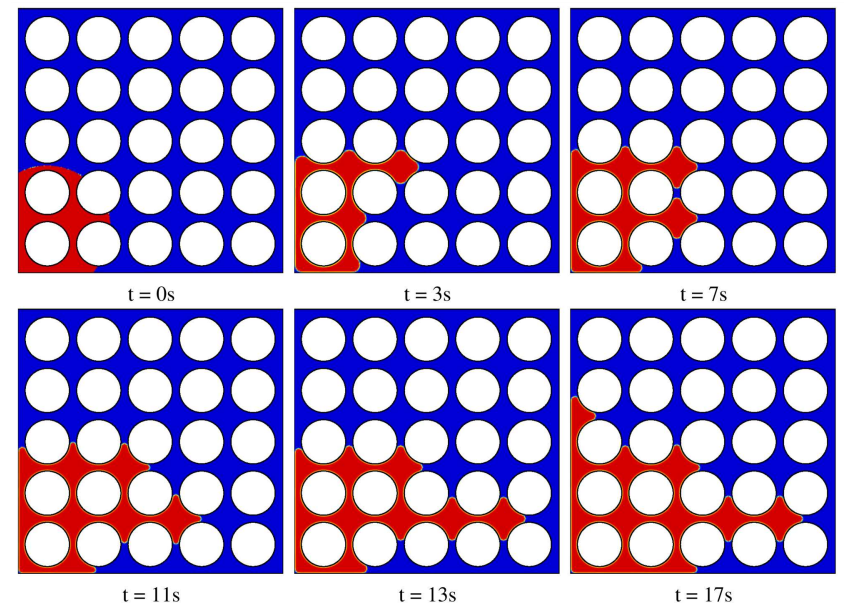

Fig. 1. Freezing simulations within the porous medium. The rectangular domain consists of rock particles (circles) and a saturated pore (remaining area). The sample is subjected to a vertical thermal gradient. The boundaries are fixed in the normal directions except for the top boundary. The left boundary is completely fixed. Freezing was initiated in the left bottom corner and spread within the pore between the particles (void circles). Red color denotes solid phase, and blue color denotes liquid phase. 

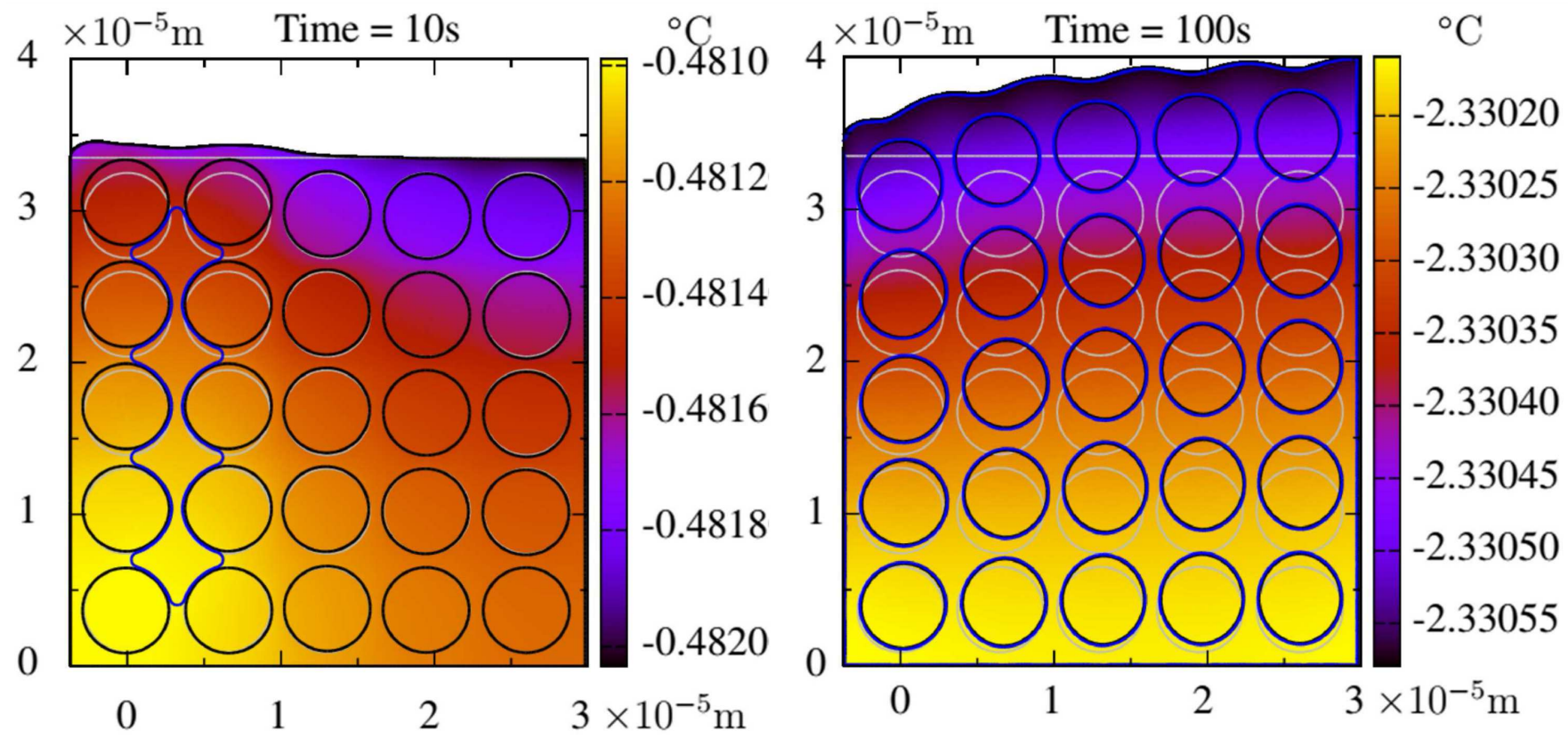

Fig. 2. Mechanical response - heaving of the free sample boundary. Freezing was initiated in the left part of the domain (blue contour) and gradually spread over the whole pore. Color signifies the current (time in s) distribution of the temperature in ${ }^{\circ} \mathrm{C}$ within the sample.

Figure 1 shows that in spite of the uniform ambient conditions and the symmetric sample structure, the evolution of the solid phase evinces a spreading pattern, which seems to be quite different from what one could expect in a case of a pure substance solidification or even of a species population spreading.

The evolution pattern in Fig. 1 stems from a direct process of following the current energy-preferential pathways. These are created by the joint effect of gradual but, due to the new phase domain evolution, spatially changing sample cooling and of the local potential of new interface shape creation provoked by the sample configuration.

In Fig. 2, the structural response (magnified by factor 50 ) of the sample just with a different initial spread of the solidifying phase is depicted in two instances of the cooling. Here the current temperature fields are revealed as well in order to indicate the current preferential heat directions affecting the new phase pathways as mentioned above.

The results have been obtained using the finite-element method and BDF time-solver implemented by the PDE solver package of COMSOL Multiphysics software. The numerical discretization has been performed employing the quadratic Lagrange elements on a mesh consisting, for the presented example, of 38,366 triangular elements, which has resulted into 333,327 degrees of freedom to solve.

\section{Conclusion}

The presented model has abilities to provide qualitative and quantitative results as shown in the previous section. It allows to obtain the dynamics of the thermomechanical interaction during phase transitions at the micro-scale and is intended to provide preliminary results in an effort to broaden the knowledge of freezing and thawing processes in porous media. The presented computation has been obtained on an ideal grain grid domain. However, it can be applied within a general geometry configuration as well.

\section{Acknowledgments}

The research has been partly supported by the project of the Czech Science Foundation (No. 14-36566G).

\section{References}

[1] Transport Phenomena in Porous Media III, Eds. D.B. Ingham, I. Pop, Elsevier, Amsterdam 2005.

[2] D. Michel, B.F. Borisov, E.V. Charnaya, W.-D. Hoffmann, P.G. Plotnikov, Yu.A. Kumzerov, Nanostruct. Mater. 12, 515 (1999).

[3] P. Jany, A. Bejan, Report DU-AB-6, Dep. Mech. Eng. and Mater. Sci., Duke University, Durham 1987.

[4] R.R. Gilpin, Water Resour. Res. 16, 918 (1980).

[5] A.C. Fowler, SIAM J. Appl. Math. 49, 991 (1989).

[6] J. Hartikainen, M. Mikkola, in: Proc. of the 6th Finnish Mechanics Days, Eds. J. Aalto, T. Salmi, University of Oulu, Oulu 1997, p. 1.

[7] R.L. Michalowski, Cold. Reg. Sci. Technol. 22, 47 (1993).

[8] R.D. Miller, in: Proc. 3rd Int. Conf. on Permafrost, National Research Council of Canada, Edmonton 1978, p. 707.

[9] A. Žák, M. Beneš, T.H. Illangasekare, IAENG Int. J. Appl. Math. 43, 127 (2013). 
[10] A. Žák, M. Beneš, T. H. Illangasekare, in Proc. Algoritmy 2016, 20th Conf. on Sci. Comp., Eds. A. Handlovičová, D. Ševčovič, Publishing House of Slovak University of Technology in Bratislava, Bratislava 2016, p. 234.

[11] A. Žák, M. Beneš, T.H. Illangasekare, A.C. Trautz, Commun. Comput. Phys. 24, 557 (2018).

[12] J.W. Gibbs, in: Collected Works, Vol. I, Thermodynamics, Longmans, Green, New York 1928, Ch. III.

[13] A. Mikelić, M.F. Wheeler, Math. Mod. Meth. Appl. Sci. 22, 1 (2012).

[14] M.E. Gurtin, I. Murdoch, Arch. Rational Mech. Anal. 57, $291(1975)$.

[15] M.E. Gurtin, A. Struthers, Arch. Rational Mech. Anal. 112, 97 (1990).

[16] S. Davis, in: Perspectives in Fluid Dynamics, Eds. G. Batchelor, H. Moffatt, M. Worster, Cambridge University Press, Cambridge 2002, p. 1.
[17] M.G. Worster, in Ref. [16], p. 393.

[18] M.E. Gurtin, Arch. Ration. Mech. Anal. 96, 199 (1986).

[19] J.R. King, J.D. Evans, SIAM J. Appl. Math. 65, 1677 (2005).

[20] J.W. Cahn, J.E. Hilliard, J. Chem. Phys. 28, 258 (1958).

[21] S.M. Allen, J.W. Cahn, Acta Metall. 27, 1084 (1979).

[22] G. Caginalp, IMA J. Appl. Math. 44, 77 (1990).

[23] M. Beneš, Interfaces Free Bound. 3, 201 (2001).

[24] M. Beneš, Acta Math. Universitatis Comenianae 70 123 (2001).

[25] A.W. Rempel, J.S. Wettlaufer, M.G. Worster, Phys. Rev. Lett. 87, 088501 (2001). 